\title{
Odour representation in honeybee olfactory glomeruli shows slow temporal dynamics: an optical recording study using a voltage- sensitive dye
}

\author{
C. Giovanni Galizia *, Armin Küttner, Jasdan Joerges, Randolf Menzel \\ Institut für Neurobiologie, Fachbereich Biologie, Freie Universität Berlin, Königin Luise Str. 28-30, 14195 Berlin, Germany
}

Received 8 July 1999; accepted 5 September 1999

\begin{abstract}
Stimulation with odours has been shown to elicit characteristic patterns of activated glomeruli in the antennal lobe (AL) of honeybees. In this study we show that these patterns are dynamic in a time window of 2-3 s after stimulus onset. We measured changes in the averaged membrane potential of all cells in the glomerular neuropil by optical imaging of the voltage-sensitive dye RH795 using a slow scan CCD camera (3 frames/s). The four substances 1-hexanol, hexanal, citral and clove-oil as well as the binary mixtures hexanol+hexanal and hexanol+citral were used as stimuli (2 s stimulus duration). We found that: (1) every odour elicited an odour-specific activity pattern, and conversely every glomerulus had a characteristic odour response profile; (2) some glomeruli had a tonic, some a phasic-tonic, and some a slow phasic response pattern; (3) the difference between the glomerular response patterns increased within $2 \mathrm{~s}$ of stimulus presentation, which suggests that odour representations became more characteristic over stimulus time; and (4) the responses to odorant mixtures were complex and glomerulus-dependent: some responses correspond to the sum of the compounds' responses, some to the response of one of the components. C) 2000 Elsevier Science Ltd. All rights reserved.
\end{abstract}

Keywords: Antennal lobe; Membrane potential; Olfactory coding; Apis mellifera

\section{Introduction}

The antennal lobe (AL) of insects is the functional analogue of the olfactory bulb in mammals, and the first central neuropil where information from the olfactory receptor neurons $(\mathrm{ORN})$ is processed. In the honeybee, there are 60,000 ORNs on each antenna (Esslen and Kaissling, 1976) which project to the roughly 160 glomeruli of the AL (Arnold et al., 1985; Flanagan and Mercer, 1989a; Galizia et al., 1999a). Here, the incoming information is processed by about 4000 local interneurons (Witthöft, 1967), and then relayed to higher order brain centres by 800 projection neurons (Hammer, 1997).

Each ORN appears to have a broad though characteristic molecular response profile, so that it responds to a

* Corresponding author. Tel.: +49-30-838-6454; fax: +49-30-8385455.

E-mail address: galizia@zedat.fu-berlin.de (C.G. Galizia). particular set of odorant molecules (Vareschi, 1971; Akers and Getz, 1993). The responses of ORNs in honeybees generally follow the stimulus duration in a phasic-tonic manner (Akers and Getz, 1993; Lemon and Getz, 1997), but recordings from interneurons and projection neurons show a much wider array of response patterns, with excitatory and inhibitory responses, late responses, patterned responses and complex responses (Fonta et al., 1991; Sun et al., 1993; Flanagan and Mercer, 1989b; Abel, 1997). These temporal response properties and the timing of spikes in these neurons are an important part of the olfactory code (Laurent, 1996). It is unclear how the different neurons involved, with their different connectivity patterns, temporal properties and response profiles to odours, extract the behaviourally important information from odourous stimuli.

Optical recording techniques have been successfully applied in order to visualise odour-evoked activity patterns in olfactory neuropils of different animals (Delaney et al., 1994; Kleinfeld et al., 1994; Cinelli et al., 1995; Friedrich and Korsching, 1997, 1998). Measurements in 
honeybees using calcium-sensitive dyes have shown that odours elicit specific spatial activity patterns (Joerges et al., 1997), that these patterns are bilaterally symmetric (Galizia et al., 1998), and that the spatial glomerular odour code is conserved within Apis mellifera (Galizia et al., 1999b).

However, calcium signals are not very suitable for temporal analyses because of their generally slow decaying phase. We therefore measured odour-evoked activity patterns in the honeybee AL using the fast voltage-sensitive dye RH795, which allowed us to monitor overall membrane depolarisation in the honeybee AL glomeruli as a response to stimulation with pure odorants or with binary mixtures of odorants. With this method we could follow the changes of activity in the peristimulus time window, showing that spatial odour representation is sharpened within this time interval.

\section{Materials and methods}

\subsection{Optical recording}

Optical recording was performed as described elsewhere (Galizia et al., 1997). Briefly, free-flying bees were collected from the hive, chilled in order to anaesthetise them and fixed in a metal tube. A window was cut into the head capsule and the brain was exposed by removing glands and tracheae. Then the head was cut off and was placed in an incubating chamber containing $200 \mu \mathrm{l}$ of a $20 \mu \mathrm{M}$ solution of RH795 (Molecular Probes, Eugene) in Ringer (130 mM NaCl, $6 \mathrm{mM} \mathrm{KCl,} 4 \mathrm{mM}$ $\mathrm{MgCl}_{2}, 5 \mathrm{mM} \mathrm{CaCl}_{2}, 160 \mathrm{mM}$ sucrose, $25 \mathrm{mM}$ glucose, $10 \mathrm{mM}$ HEPES, pH 6.7, $500 \mathrm{mOsmol}$ ). After $40 \mathrm{~min}$ incubation at $10^{\circ} \mathrm{C}$, the head was fixed with wax in a recording chamber, the brain was covered with a coverslip, and the recording chamber fixed under the microscope, with constant Ringer perfusion (composition as above). At all times, great care was taken to keep both antennae dry and moving freely in the air.

Sequential images where recorded under an upright microscope (Zeiss Axioskop, with a 40× NA 0.6 objective) under epifluorescent illumination (filter set BP546 for excitation, 580 dichroic, LP 590 fluorescence) with a slow-scan 12 bit CCD camera (Photometrics, CH250A, Tucson, AZ). The light source was a halogen lamp (100 W, Philips 64458AX) driven by a stabilised power supply (68830, Lot Oriel, Darmstadt, Germany). Scanning frequency was 3 frames/s, exposure time for each frame being between 50 and $200 \mathrm{~ms}$. Each measurement consisted of 40 frames. Either an odour stimulus or the odourless control was applied between the 9th and the 13th frame. Interstimulus interval was 1 min. Odour stimulation was computer-controlled, using an olfactometer with separate channels for each odour
(Galizia et al., 1997). This olfactometer switched from a continuous blank to an odour-laden air stream, avoiding any mechano-sensory component to the signals. Odour stimuli were prepared by applying $4 \mu \mathrm{l}$ pure substance onto a filter paper, and placing the filter paper in a $1 \mathrm{ml}$ plastic syringe. The syringe was cut to the length of $7 \mathrm{~cm}(0.7 \mathrm{ml})$ in order to fit into the olfactometer. The resulting stimulus concentrations are nonsaturating and routinely used in the lab for behavioural experiments. Binary odorant mixtures were given by simultaneously opening two odorant channels of the olfactometer, so that the mixed stimulus contained each component at approximately half concentration. The odours used were 1-hexanol, hexanal, citral (Sigma, Deisenhofen, Germany) or clove-oil (local drug store). These substances are all common components of plant odours. In addition, citral is also a component of the pheromone produced in the Nasanov gland. However, there is no special sub-compartment for citral or other non-sexual pheromones in the area of the AL which is optically accessible in this study (Joerges et al., 1997; Galizia et al., 1999b).

We saw only weak signals (the strongest responses were in the range of $1 \% \Delta F / F$, but compare Figs. 1-3, with some signals below $0.1 \%$ ) which made it necessary to average between 8 and 16 stimulations for one measurement in order to improve the signal/noise $(\mathrm{S} / \mathrm{N})$ ratio. This is partly due to the relatively high background fluorescence of cells not contributing to the signal, most likely of cell membranes which are not depolarised by the stimulus (glia cells, epithelium, somata, nonexcited neurons).

The experiment was controlled by a mainframe OS9 computer (Eltec Eurocom-6). Further data processing was done on Macintosh computers using custom-made software written in IDL Language (Research Systems, Boulder, $\mathrm{CO}$ ).

\subsection{Signal evaluation}

Signals were calculated from the raw fluorescent data as $\Delta F / F . F$ was taken as the average of five frames before stimulus onset.

Signals were evaluated in the following way: in the image of the $\mathrm{AL}$, as obtained during the measurements, the borders of the glomeruli could be recognized (see Fig. 1). The time-course for each glomerulus was averaged over its area (thus improving the $\mathrm{S} / \mathrm{N}$ ratio), producing curves as shown in Fig. 2. For quantitative evaluation, the control response to a blank stimulus (air current) was subtracted from the response. This procedure corrected for bleaching. In order to analyze the dynamic component of odour responses, signal intensities were evaluated from the bleach-corrected timecourses at three times (Fig. 4A): $300 \mathrm{~ms}$ after stimulus onset (time T); $2000 \mathrm{~ms}$ after stimulus onset, coinciding 
with stimulus offset (time $T^{\prime}$ ); and $2600 \mathrm{~ms}$ after stimulus onset (time $T^{\prime \prime}$, corresponding to $600 \mathrm{~ms}$ after the end of the stimulus). A total of $n=6$ bees were evaluated in this way.

Each response at any of the three times was described as a vector in a space defined by the dimensions equal to the number of glomeruli recognized, where each element of the vector represents the response in a glomerulus (Schild and Riedel, 1992; Shepherd, 1993). The dynamic component of the response pattern to each odour is thus represented in the sequence of three vectors (one for each of the times $T, T^{\prime}$ and $T^{\prime \prime}$ ). The similarity of the representation of two odours was calculated as the scalar product of two such vectors. By correcting for the absolute size of the response, the angle between these vectors could be obtained. This allows for the comparison of the spatial activity pattern without any influence from the exitation strength. The similarity between odour representations was quantified in this way for comparing different times of the same odour response, and for comparing responses to different odours. Statistical analysis was carried out using the program JMP 3.2.1 (SAS, Cary, NC) on a Macintosh computer.

\section{Results}

\subsection{Spatial characteristics of odour-evoked activity patterns}

Stimulation with odours gives spatio-temporal response patterns in the AL of the honeybee (Fig. 1A). With stimulus onset, wide areas in the AL increase their mean depolarisation, with individual glomeruli being particularly strongly activated. After stimulus offset, activity declines rapidly. The staining with the voltagesensitive fluorescent dye RH795 reveals the compartmentalisation of the AL into glomeruli. In the images, the positions of olfactory glomeruli could be identified (Fig. 1B,C), because the borders appeared brighter than the centres. Activity foci correspond to olfactory glomeruli (Fig. 1B), as can be seen by superimposing the glomerular layout onto the false-colour coded activity map. It was possible to discriminate up to 20 glomeruli. The response of each glomerulus was integrated to increase the signal-to-noise ratio. However, the images were not of sufficient quality to identify the glomeruli using an atlas of the AL (Galizia et al., 1999a), and therefore the data could not be compared between individuals at the level of individual glomeruli.

In all ALs we found glomerular responses to each of the four odours tested (hexanol, hexanal, citral and clove-oil) and to their binary mixtures. When integrating over the entire stimulus duration, individual glomeruli showed different response intensities (Fig. 1D): some glomeruli did not respond at all (e.g. glomerulus 4); some responded to only one odour (e.g. glomerulus 7); others responded to a few; and some to all four odours (see pie charts in Fig. 1D). The glomeruli differed with respect to their overall response strength and the complex response pattern integrated over all glomeruli (i.e. the response vector) was specific for each odour.

\subsection{Temporal characteristics of odour-evoked activity patterns}

Examples for the time-course of the signals are shown in Fig. 2, for six different glomeruli. Due to the dye and the filter settings, membrane depolarisations lead to negative signals (downward deflections of the curves). Signal size $(\Delta F / F)$ is between 0.1 and $0.6 \%$, and differs both between glomeruli and for different odours. Bleaching was around $0.2 \%$ over the time of each measurement (12 s). Note that the dotted line, the air control, does not show any signal apart from the bleaching, which is identical to the bleaching in the measurements with an odorant stimulus. This allowed us to subtract the air control in the quantitative analysis and thus correct for the bleaching effect.

The time-course is different for different odours, as can be seen in Fig. 2E, where hexanal elicits the strongest response, but quickly decays after stimulus-offset, while citral, with a smaller signal at stimulus-onset, has a slower decay time. We therefore investigated how these characteristic time-courses influence odour representation in the AL.

In Fig. 3 we superimposed different response traces, categorizing them according to their shapes. Responses with clear tonic behaviour and sharp off-set are displayed in Fig. 3A: the signal is strong during the stimulus, and decays rapidly at the end of the stimulus. Curves in Fig. 3B have a phasic-tonic pattern: again, onset is rapid, but the signals slowly decay while the stimulus is on. Furthermore, upon stimulus offset, the signals often decay only slowly. Responses collected in Fig. 3C have a phasic shape: onset is rapid, but the signals immediately begin to decay, and almost reach baseline levels by the end of the stimulus. A comparison with Fig. 2 shows that these are not glomerulus-specific properties: the same glomerulus may respond with a tonic signal to one odour, and with a phasic shape to another. In all cases, however, signal onset was very rapid.

\subsection{Comparing mean activity: the signal decreases in time}

A corollary of these observations is that the total signal decreases during stimulus presentation and after stimulus offset. Furthermore, since the dynamic properties are both glomerulus- and odour-dependent, the relative activity of the glomeruli may change in time, which would imply a shift in the glomerular activity pattern. 
A
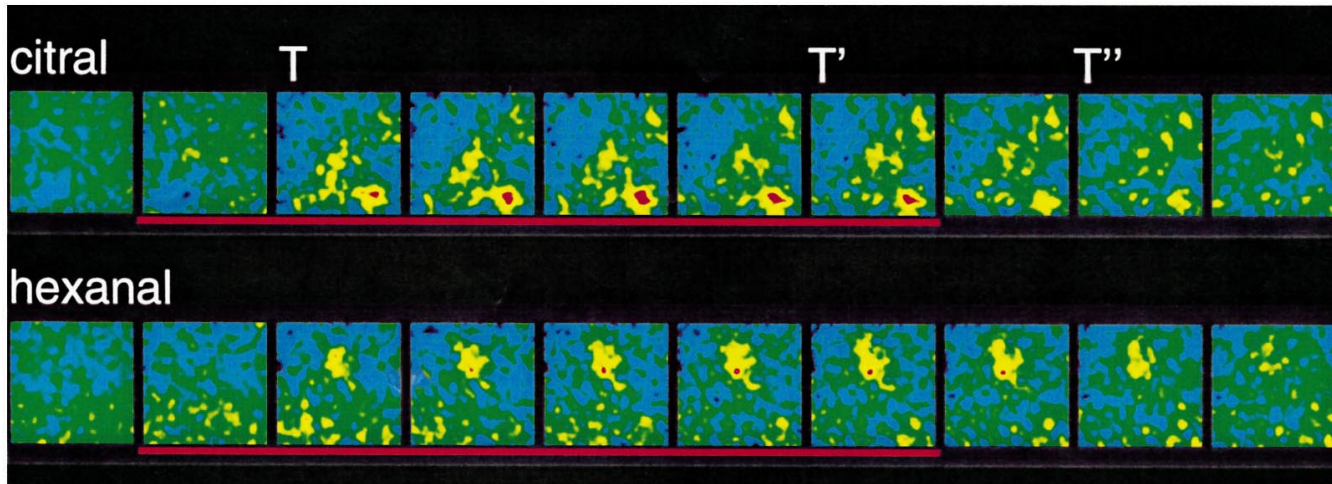

$-0.08 \Delta F / F \square 0.05 \Delta F / F$
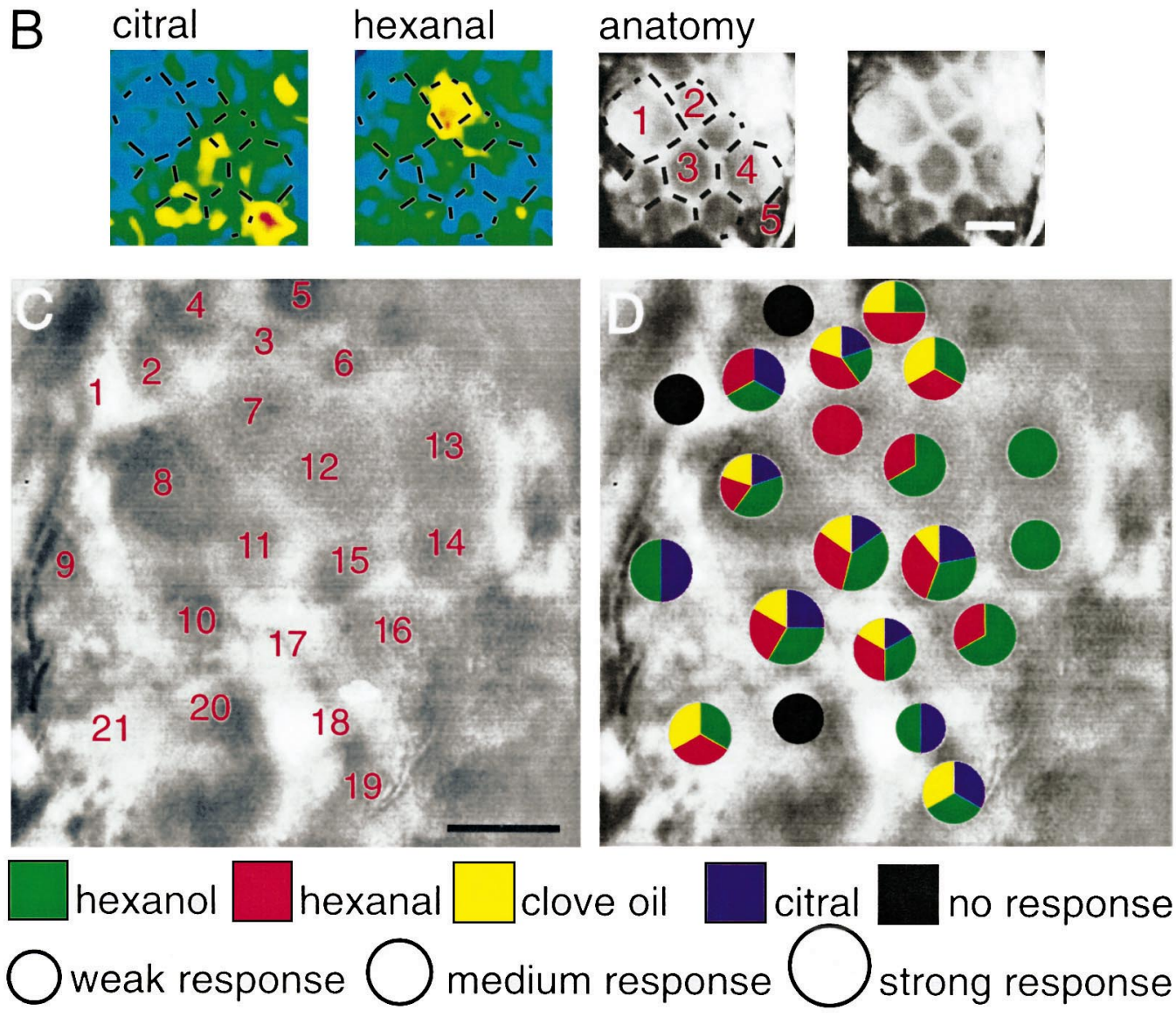

Fig. 1. (A) False-colour coded representation of a response to citral and a response to hexanal, corrected for bleaching by subtracting the response to air control. Each image is spatially filtered, no temporal filter has been applied. Scaling is equal for the two series. The false-colour code is given by the bar at the bottom. Duration between frames is $333 \mathrm{~ms}$. Red bar gives stimulus delivery ( $2 \mathrm{~s})$, the three time-points considered in the subsequent analysis are indicated with $T, T^{\prime}$ and $T^{\prime \prime}$. (B) Single-frame representation of the response to citral and hexanal (same animal as in A). Superimposed are the borders of glomeruli, as taken from the anatomical view to the right. The labelling of glomeruli is arbitrary. For example, glomerulus 1 does not respond to citral, but very weakly to hexanal; glomerulus 2 responds strongly to hexanal, but not to citral; the response in glomerulus 3 is stronger to citral than to hexanal; and glomerulus 5 is very strongly activated by citral. Same colour code as in A. Note the correspondence of physiological response with morphological borders of the glomeruli, which is evident in glomeruli with strong responses, i.e. glomerulus 5 for citral and glomerulus 2 for hexanal. Scale bar $50 \mu \mathrm{m}$. (C) Fluorescent photograph of an antennal lobe stained with RH795. The glomeruli can clearly be seen. Twenty-one glomeruli have been labelled with arbitrary numbers; these numbers do not refer to those used in the standard atlas of the honeybee antennal lobe (Galizia et al., 1999a). Different animal from A and B. Scale bar $50 \mu \mathrm{m}$. (D) Same image as in C, with superimposed stimulus-response profiles. The relative response intensity to the four tested odours is given by the relative portion of the pie chart. Black circles indicate glomeruli that did not respond to any of the tested odours. The overall response over stimulus time was evaluated. Bigger circles indicate glomeruli with overall larger responses (three size classes). Note that some glomeruli were quite selective: e.g. nos 13 and 14 for hexanol, no. 7 for hexanal, while others responded to two, three or even all four components (e.g. glomerulus 3 ). 

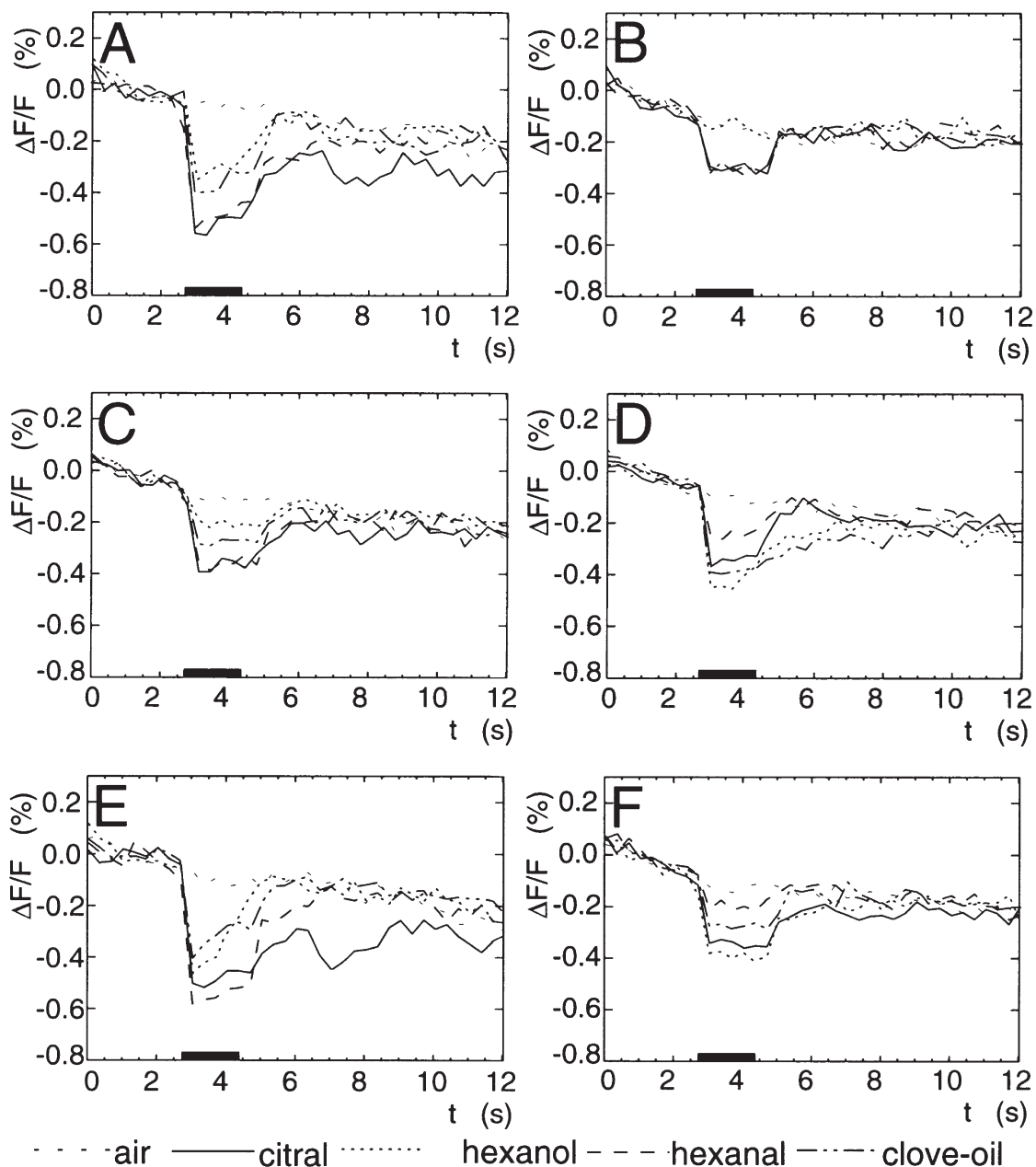

Fig. 2. Time courses of responses to different odours for six different glomeruli, A-F. Change in relative fluorescence is plotted against time (s). A negative signal indicates increased membrane depolarisation. The black bar indicates the stimulus. Note the overall bleaching of around $0.2 \%$ during the measurements, and the lack of response to the air control stimulus in all glomeruli. Note also the different shape properties of the responses (see text for detailed discussion). No filter was applied to the data.

In order to investigate this we calculated the response pattern for the different phases of the response $\left(T, T^{\prime}\right.$ and $T^{\prime \prime}$ in Fig. 4A). The mean signal intensity for these three intervals (all odours and glomeruli averaged) is shown in Fig. 4B: immediately after stimulation, response intensity varies between about $0.5 \% \Delta F / F$ and $1 \% \Delta F / F$. Response intensity decreases, and after stimulus offset mean response intensity is well below $0.5 \%$ $\Delta F / F$.

\subsection{Comparing odours: representations sharpen in time}

The representation of a particular odour is best described as a vector in a space defined by the responses of all glomeruli. This vector changes in size and direction over time for each odour. We quantified the similarity of odour representations as the angle between such vectors. In this way, similarity can be calculated without being influenced by overall response intensity. Therefore, similarity can also be compared for the different time windows $T, T^{\prime}$ and $T^{\prime \prime}$, disregarding their overall decreasing activity levels (Fig. 4B).

When comparing the mean similarity between different odour representations we found that it decreased considerably over time, from $T$ to $T^{\prime \prime}$ (see Fig. 4C). That means that, immediately upon stimulus delivery, the representations of hexanol, hexanal, citral and clove-oil were more similar than at stimulus-offset, or even later when the animal was no longer stimulated.

The same result can be described by calculating the correlation at the three times $T, T^{\prime}$ and $T^{\prime \prime}$ : the mean correlation between the glomerular activity patterns evoked by hexanol, hexanal, citral and clove-oil was 0.97 at $T$, and decreased to 0.91 at $T^{\prime}$, and 0.83 at $T^{\prime \prime}$. Therefore, odour representation becomes different over time.

\subsection{Comparing response phases: each odour representation moves}

We measured the degree to which the odour representation for each odour moved across the three times con- 


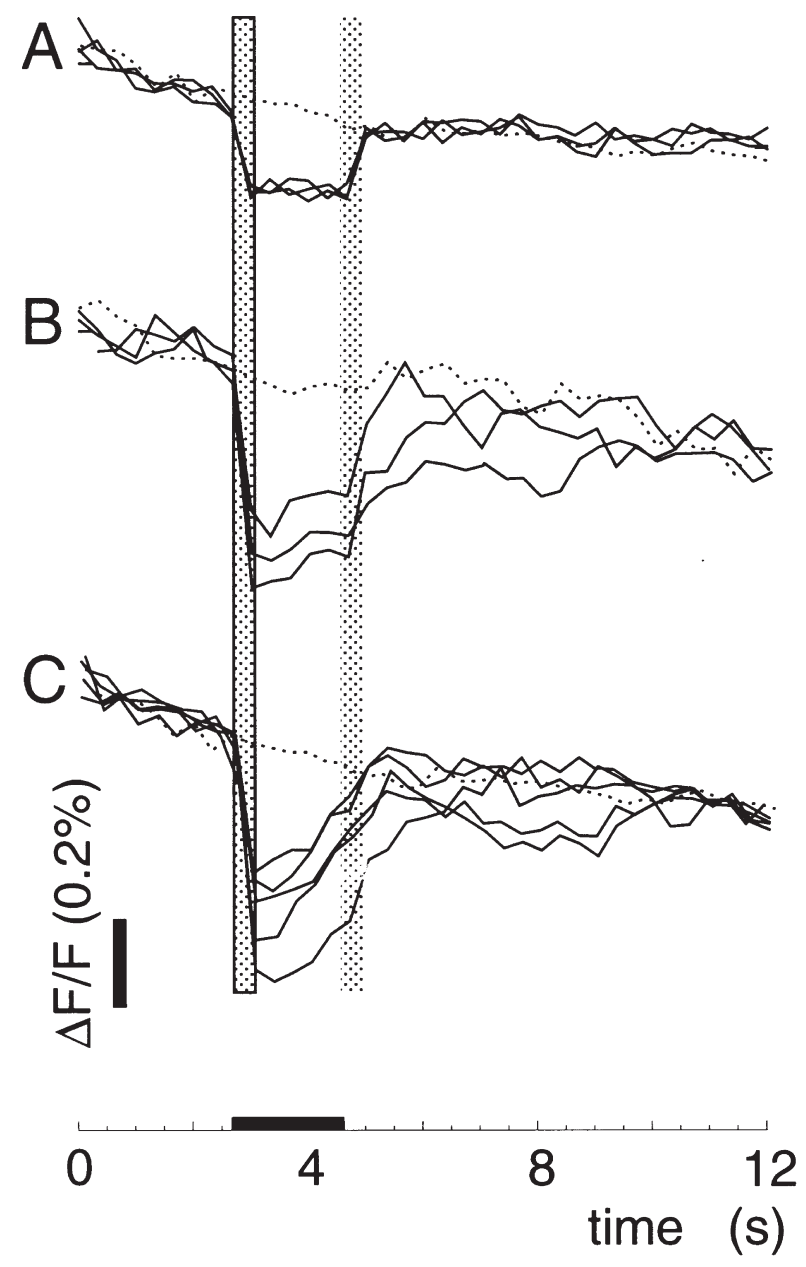

Fig. 3. Time courses of responses to odours sorted by temporal response characteristics. All responses show a fast rising flank, reaching maximum depolarisation within $300 \mathrm{~ms}$ (framed dotted box). The curves differ in their decaying phase. The plots are from various animals, glomeruli and odour stimuli, compare with Fig. 2. (A) Tonic behaviour: upon stimulus end, the membrane potential readily returns to baseline levels. (B) Phasic-tonic behaviour. (C) Phasic characteristic: the signal gradually decays within $2-3 \mathrm{~s}$ after stimulus onset.

sidered. The result was quite surprising, and is shown in Fig. 4D: the mean shift $\phi$ between $T$ and $T^{\prime}$ comprised an angle of around $\phi=25^{\circ}$, which corresponds to $\cos (\phi)=0.9$. This implies that the representation of an odour is slightly different at $T^{\prime}$ as compared to $T$. This shift greatly increased between $T^{\prime}$ and $T^{\prime \prime}$, with an angular movement of about $\phi=40^{\circ}$, corresponding to $\cos (\phi)=0.75$. However, when we compared the vectors at $T^{\prime \prime}$ with the initial vectors at $T$, we found that the overall vector travelled was again in the range of $\phi=25^{\circ}$, i.e. about $\cos (\phi)=0.9$. Therefore, the activity vector moves during odour presentation, but does not travel far away from the initial pattern.

\subsection{The responses to odorant mixtures}

In addition to the single odour stimuli we tested the responses to the two binary odour mixtures, hexanol+hexanal and hexanol+citral. When comparing responses to binary stimuli with those to single components we found differences among glomeruli. Character-
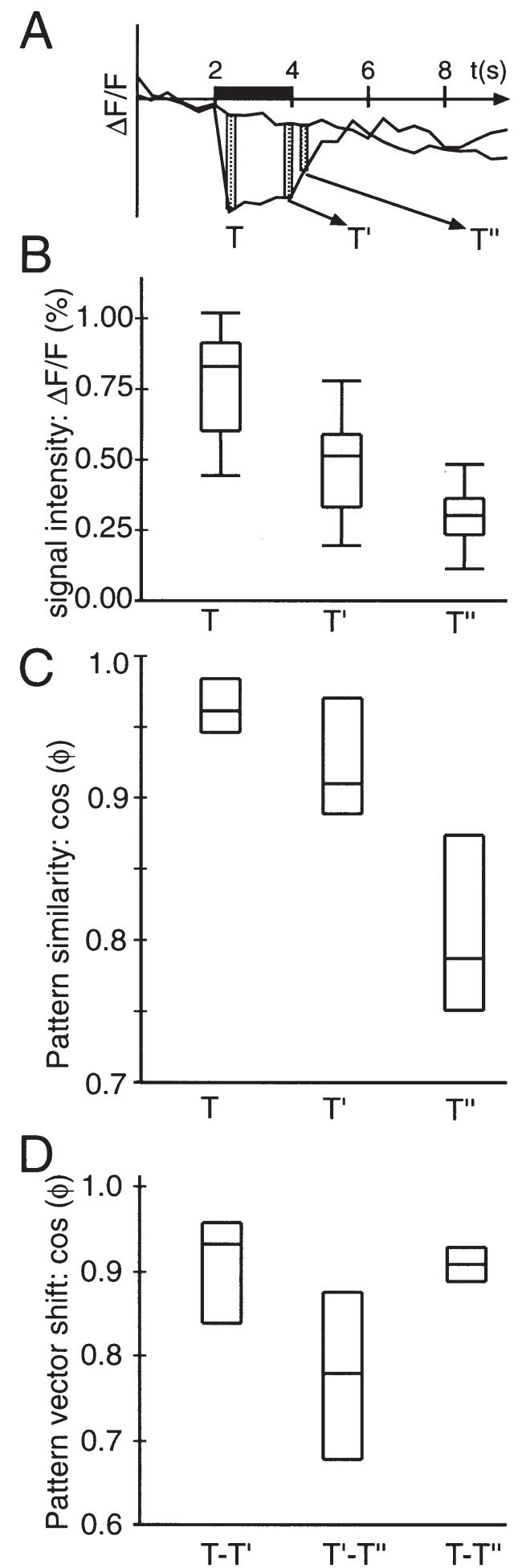
istic response types are shown in Fig. 5: some glomeruli respond to the mixture with a signal corresponding to the sum of the two components' responses (Fig. 5A,B), some with an intermediate response (Fig. 5E), and some with a response identical to just one component, as in Figs $5 \mathrm{C}$ and $\mathrm{D}$, where one of the two components is ineffective, and Fig. 5F, where the response to the mixture is almost identical to that elicited by a single component.

\section{Discussion}

In this paper we show that the glomerular activity pattern in the AL of honeybees is a dynamic process in the time domain of a few seconds. In honeybees' ALs, odours are thus represented as a dynamical spatial pattern of glomerulus excitation. We show that odour representation becomes more different within 2 s upon stimulus delivery, and thus more characteristic for the particular odour, which suggests that odour discriminability should also increase in this time window.

\subsection{The optical signal}

In measurements of intracellular calcium response onset is generally fast, but return to baseline is slow, leading to a superposition when, in an ensemble response, more cells join the previously active cells. Optically measuring membrane depolarisation with voltage sensitive dyes allows a better temporal resolution than calcium imaging. However, since we bath applied

Fig. 4. Statistical analysis of the temporal changes in odour representation. (A) Schematic of the three time-windows compared: $T$ corresponds to the maximum initial response ( $300 \mathrm{~ms}$ after stimulus onset), $T^{\prime}$ corresponds to $2000 \mathrm{~ms}$ after stimulus onset, and $T^{\prime \prime} 2600 \mathrm{~ms}$ after stimulus onset. (B) Overall response intensity decreases from $T$ to $T^{\prime}$ and $T^{\prime \prime}$. Intensities for all measured odour responses averaged over all glomeruli have been considered ( $n=24$ mean values from four odours tested in six bees). The box plots give the median, the 25 and $75 \%$ quantiles in the box and the 10 and $90 \%$ quantiles of the observed values. (C) The similarity between the patterns for different odours decreases within $2 \mathrm{~s}$. Each glomerular response was represented as a vector in a space defined by the responses of each glomerulus, and the angles between the representations of different odours were calculated from these vectors. $n=36$ for six binary comparisons of four odours in six preparations. The box plot gives the median and the 10 and $90 \%$ quantiles of the calculated angles at times $T, T^{\prime}$ and $T^{\prime \prime}$. A $\cos (\phi)$ value close to 1 means very similar representation, a value of $\cos (\phi)=0$ would indicate two linearly independent vectors. (D) Odour representation shifts in time. Here the vector representations between time $T$ and $T^{\prime}$, time $T^{\prime}$ and $T^{\prime \prime}$, and $T$ and $T^{\prime \prime}$ have been compared for each odour. A value of $\cos (\phi)=1$ would indicate no change in vector direction. Note that there is a strong shift between $T^{\prime}$ and $T^{\prime \prime}$, but that this shift does not lead the representation farther away from the initial place in the multidimensional glomerular space, because the shift from $T$ to $T^{\prime \prime}$ is almost equal to that from $T$ to $T^{\prime}$. Median, 10 and $90 \%$ quantiles are given. the dye, the resulting signal is a composite of different cell types.

Every glomerulus receives-on average-input from about 375 ORN axons [assuming 60,000 ORNs altogether (Esslen and Kaissling, 1976) and 160 glomeruli (Flanagan and Mercer, 1989a; Galizia et al., 1999a)], all of which profusely branch in the outer layer of the glomerulus. Furthermore, every glomerulus is visited on average by collaterals of about 1300 local interneurons, and densely impregnated by 20 local interneurons and five projection neurons. All of these contribute to the measured signal.

Which cells are active at times $T, T^{\prime}$ and $T^{\prime \prime}$ ? Most ORN recordings show a time-course which mimics the stimulus duration, and end their activity at stimulus offset (Akers and Getz, 1993), and are therefore strongly active at times $T$ and $T^{\prime}$. In single cell recordings of local interneurons and projection neurons, an excitation outlasting stimulus duration has often been observed (Flanagan and Mercer, 1989b; Fonta et al., 1991; Sun et al., 1993; Abel, 1997). In most glomeruli we see an activity tail at $T^{\prime \prime}$ after stimulus offset (Fig. 3B,C).

Still, all three response phases observed may contain signals from ORNs, local interneurons and projection neurons. Furthermore, since the proportion of membranes stained is unknown, no quantitative evaluation of these signal components can be attempted. Pharmacological treatments and electrophysiological recordings (e.g. extracellular nerve recordings) have to be added in order to quantitatively clarify their relative contribution. Nevertheless, it is likely that the first response $(T)$ contains a strong (though not quantifiable) component due to afferent ORNs, that the intermediate time window $\left(T^{\prime}\right)$ reflects ORNs, local interneurons and projection neurons, and that the late component $\left(T^{\prime \prime}\right)$ is predominantly due to interneurons and projection neurons.

\subsection{Plasticity in the olfactory code}

Odour representation is optimized during stimulus delivery. When stimulating with an odour, the representation at the beginning of the odour is different from the representation at the end of that stimulation, and comparing these final representations between the odours tested shows a greater difference than at the beginning of stimulus delivery. In recent experiments Stopfer and collegues showed that repeated presentation of odours changes the oscillatory behaviour in the field potential of the locust brain. In particular, no oscillatory activity was observed at the very first odour presentation, while after repeated odour presentation spike timing of projection neurons increased in precision (Stopfer and Laurent, 1999). In our experiments, we had to average at least eight stimuli for each measurement. Therefore, the animal experienced both odours repeatedly. Furthermore, stimulus delivery of the different odours was interlaced. 

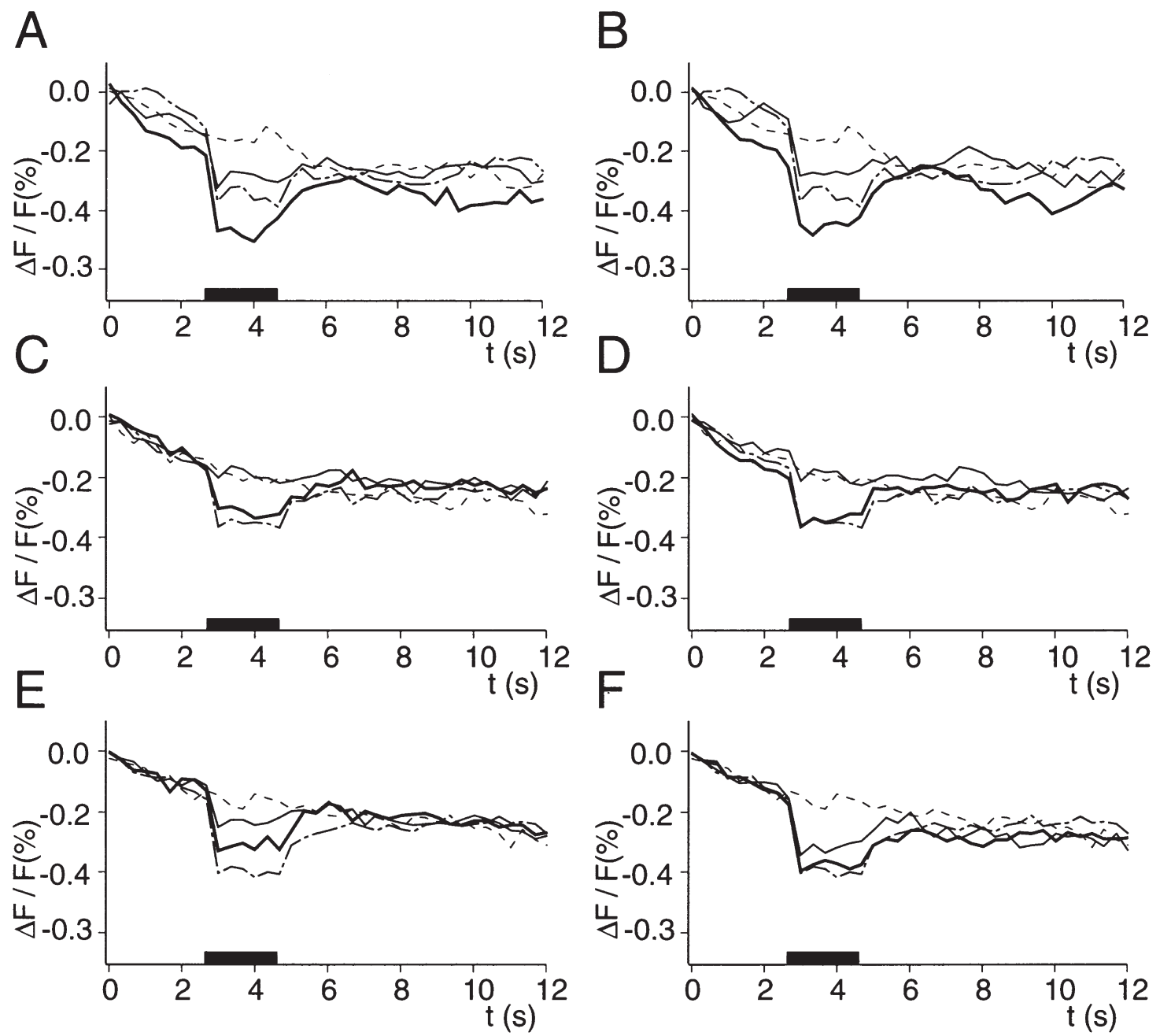

Fig. 5. Time courses of responses to single odours compared to the responses to binary mixtures. Each row (A,B and C,D and E,F) represents a glomerulus. The columns (A,C,E and B,D,F) represent the responses to two different odour mixtures: A, C, E (left column) show the responses to citral (thin line), hexanol (dash-dot) and their mixture (thick line), while B, D, E (right column) show the responses to hexanal (thin line), hexanol (dash-dot) and their mixture (thick line). In all traces, the dashed line represents the response to air control. Note that in A and B the response to the mixture roughly corresponds to the sum of the components' response. In $\mathrm{C}$ and $\mathrm{D}$ the mixture elicits the same response as one component (in these cases, the other component neither elicited a response, nor did it influence the response to the mixture). In $\mathrm{E}$ the response to the mixture is intermediate to the components' responses. In F it corresponds to the stronger component.

Consequently, it may be that the bee optimised its $\mathrm{AL}$ network during the experiment in order to distinguish the odours which it was repeatedly experiencing. We do not know whether already for the first odour presentation response patterns are more different between odours at the end of the $2 \mathrm{~s}$ stimulus time, as compared to stimulus onset. It should be possible to test this hypothesis by using an experimental set-up having a better $\mathrm{S} / \mathrm{N}$ ratio, thus permitting single trial measurements of voltagesensitive dyes. The capability of the AL to change its activity patterns following learning has been shown in optical measurements where mapping the glomerular activity pattern was combined with appetitive olfactory conditioning (Faber et al., 1999).

\subsection{Dynamic olfactory coding in the $A L$}

The glomerular activity pattern has been shown to be odour-specific: each odour elicits a particular mosaic of active glomeruli, and each glomerulus plays a part in the pattern for different odours (Joerges et al., 1997). These results were obtained with calcium imaging, and reflect the spatial component of the olfactory code. Using electrophysiological measurements, Laurent and co-workers found characteristic oscillations in the range of 30-40 $\mathrm{Hz}$, and suggest that these fast events are important for olfactory coding (Stopfer et al., 1997). Stimulation with odours has been shown to lead to evolving neural assemblies in the AL (Laurent, 1996), which correspond 
to the intermediate time window, in the range of 2-3 s as analysed in this paper, in which the representation of odours changes. Furthermore, Stopfer and co-workers saw a lack of behavioural discrimination between octanol and hexanol in honeybees after picrotoxin (PTX) superfusion of the brain, but no deficit in the distinction between less similar odours (Stopfer et al., 1997). This suggests that the inhibitory network of the AL is important for fine-tuned recognition of odours. Most likely, our finding that the spatial odour representation becomes more unique within $2 \mathrm{~s}$ is also accomplished by the inhibitory network of the AL.

These observations also suggest that the bees should recognize an odour more precisely after $2 \mathrm{~s}$ than immediately at odour presentation. Bees are able to respond to an odour as early as after $0.5 \mathrm{~s}$, but odour discrimination as tested in differential conditioning experiments is not possible for odour pulses shorter than $1 \mathrm{~s}$ (J. Klein, unpublished). It is, therefore, likely that odour identification in bees is a dynamic process allowing the bee to make better discrimination if odour stimuli are not too short and are separated over time. The time range of 1-2 $\mathrm{s}$ appears long, as compared to olfactory motor responses elicited by pheromone pulses in moths, which can be detected up to $3 \mathrm{~Hz}$ (Kaissling, 1996). However, the task for which temporal processing may be critical in bees, namely fine odour discrimination, is different from pheromone odour identification. It is likely that finely-tuned odour discrimination is biologically relevant for a foraging bee only in close apposition with the food source, a flower. In such a situation, bees hovering in front of a flower take odour probes for longer durations and at lower frequencies as compared to a situation in free flight when turbulent air distributes odour plumes into small packages. Possibly, increasing the temporal resolution of the olfactory system is costly in evolutionary terms, or in competition with other properties of the AL (number of detectable and/or distinguishable odours, plasticity, etc.). Therefore, temporal resolution may be limited by the ecological necessities, and may be different for different odors.

\section{Acknowledgements}

All data analysis of this work was carried out by A. Küttner. Thanks to Dedo Koppitz for excellent workshop assistance, and to S. Sachse and M. Wurm for comments on the manuscript.

\section{References}

Abel, R., 1997. Das olfaktorische System der Honigbiene: Elektrophysiologische und morphologische Charakterisierung von Antennallobus Neuronen und deren Beteiligung beim olfaktorischen Lernen. Ph.D. Thesis, Freie Universität Berlin, Berlin.
Akers, R.P., Getz, W.M., 1993. Response of olfactory receptor neurons in honeybees to odorants and their binary mixtures. Journal of Comparative Physiology A 173, 169-185.

Arnold, G., Masson, C., Budharugsa, S., 1985. Comparative study of the antennal lobes and their afferent pathway in the worker bee and the drone (Apis mellifera). Cell and Tissue Research 242, 593-605.

Cinelli, A.R., Hamilton, K.A., Kauer, J.S., 1995. Salamander olfactory bulb neuronal activity observed by video rate, voltage-sensitive dye imaging. III. Spatial and temporal properties of responses evoked by odorant stimulation. Journal of Neurophysiology 73, 20532071.

Delaney, K.R., Gelperin, A., Fee, M.S., Flores, J.A., Gervais, R., Tank, D.W., Kleinfeld, D., 1994. Waves and stimulus-modulated dynamics in an oscillating olfactory network. Proceedings of the National Academy of Science USA 91, 669-673.

Esslen, J., Kaissling, K.-E., 1976. Zahl und Verteilung antennaler Sensillen bei der Honigbiene (Apis mellifera L.). Zoomorphology 83, 227-251.

Faber, T., Joerges, J., Menzel, R., 1999. Associative learning modifies neural representations of odors in the insect brain. Nat. Neurosci. 2, 74-78.

Flanagan, D., Mercer, A.R., 1989a. An atlas and 3-D reconstruction of the antennal lobes in the worker honey bee, Apis mellifera $\mathrm{L}$. (Hymenoptera: Apidae). International Journal of Insect Morphology and Embryology 18, 145-159.

Flanagan, D., Mercer, A.R., 1989b. Morphology and response characteristics of neurones in the deutocerebrum of the brain in the honeybee Apis mellifera. The Journal of Comparative Neurology 164, 483-494.

Fonta, C., Sun, X.J., Masson, C., 1991. Cellular analysis of odour integration in the honeybee antennal lobe. In: Goodman, L.J., Fisher, R.C. (Eds.) The Behaviour and Physiology of Bees. CAB International, Wallingford, UK, p. 227.

Friedrich, R.W., Korsching, S.I., 1997. Combinatorial and chemotopic odorant coding in the zebrafish olfactory bulb visualized by optical imaging. Neuron 18, 737-752.

Friedrich, R.W., Korsching, S.I., 1998. Chemotopic, combinatorial, and noncombinatorial odorant representations in the olfactory bulb revealed using a voltage-sensitive axon tracer. Journal of Neuroscience 18, 9977-9988.

Galizia, C.G., Joerges, J., Kuettner, A., Faber, T., Menzel, R., 1997. A semi-in-vivo preparation for optical recording of the insect brain. Journal of Neuroscience Methods 76, 61-69.

Galizia, C.G., Nägler, K., Hölldobler, B., Menzel, R., 1998. Odour coding is bilaterally symmetrical in the antennal lobes of honeybees (Apis mellifera). European Journal of Neuroscience 10, 2964-2974.

Galizia, C.G., McIlwrath, S.L., Menzel, R., 1999a. A digital threedimensional atlas of the honeybee antennal lobe based on optical sections acquired using confocal microscopy. Cell and Tissue Research 295, 383-394.

Galizia, C.G., Sachse, S., Rappert, A., Menzel, R., 1999b. The glomerular code for odor representation is species-specific in the honeybee Apis mellifera. Nature Neuroscience 2, 473-478.

Hammer, M., 1997. The neural basis of associative reward learning in honeybees. Trends in Neurosciences 20, 245-252.

Joerges, J., Küttner, A., Galizia, C.G., Menzel, R., 1997. Representations of odours and odour mixtures visualized in the honeybee brain. Nature 387, 285-288.

Kaissling, K.E., 1996. Peripheral mechanisms of pheromone reception in moths. Chemical Senses 21, 257-268.

Kleinfeld, D., Delaney, K.R., Fee, M.S., Flores, J.A., Tank, D.W., Gelperin, A., 1994. Dynamics of propagating waves in the olfactory network of a terrestrial mollusk: An electrical and optical study. Journal of Neurophysiology 72, 1402-1419.

Laurent, G., 1996. Dynamical representation of odors by oscillating and evolving neural assemblies. Trends in Neurosciences 19, 489-496. 
Lemon, W.C., Getz, W.M., 1997. Temporal resolution of general odor pulses by olfactory sensory neurons in american cockroaches. Journal of Experimental Biology 200, 1809-1819.

Schild, D., Riedel, H., 1992. Significance of glomerular compartmentalization for olfactory coding. Biophysics Journal 61, 704715 .

Shepherd, G.M., 1993. Principles of specificity and redundancy underlying the organization of the olfactory system. Microscopy Research and Technique 24, 106-112.

Stopfer, M., Bhagavan, S., Smith, B.H., Laurent, G., 1997. Impaired odour discrimination on desynchronization of odour-encoding neural assemblies. Nature 390, 70-74.
Stopfer, M., Laurent, G., 1999. Rapid and specific experience-dependent short-term changes in olfactory network dynamics enable more precise odor representation. In: Fifth IBRO World Congress of Neuroscience., p. 35.

Sun, X.-J., Fonta, C., Masson, C., 1993. Odour quality processing by bee antennal lobe interneurones. Chemical Senses 18, 355-377.

Vareschi, E., 1971. Duftunterscheidung bei der Honigbiene. Einzelzellableitungen und Verhaltensreaktionen. Zeitschrift für vergleichende Physiologie 75, 143-173.

Witthöft, W., 1967. Absolute Anzahl und Verteilung der Zellen im Hirn der Honigbiene. Zeitschrift Morphologie der Tiere 61, 160184 